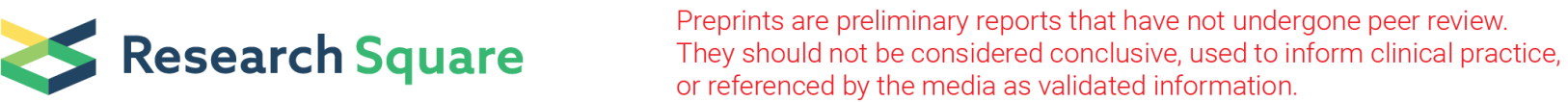

\section{Imaging Capability at Close Range of High Frequency Ultrasound Transducer on the Perforation of Bony Walls Simulating Pedicle Screw Placement}

\section{Zhiqiang Li}

Nanjing Medical University Affiliated Suzhou Hospital: Suzhou Municipal Hospital

Dongze Li

Suzhou Institute of Biomedical Engineering and Technology

\section{Suoyuan Li}

Nanjing Medical University Affiliated Suzhou Hospital: Suzhou Municipal Hospital

\section{Weiwei Shao}

Suzhou Institute of Biomedical Engineering and Technology

\section{Peiyang Li}

Suzhou Institute of Biomedical Engineering and Technology

\section{Yang Jiao}

Suzhou Institute of Biomedical Engineering and Technology

\section{Chen Yang}

Suzhou Institute of Biomedical Engineering and Technology

\section{Wenjia Lu}

Suzhou Institute of Biomedical Engineering and Technology

\section{Yaoyao Cui}

Suzhou Institute of Biomedical Engineering and Technology

\section{Junjie Fan}

Suzhou Institute of Biomedical Engineering and Technology

\section{Donglai Wang}

Nanjing Medical University Affiliated Suzhou Hospital: Suzhou Municipal Hospital

Tianming Zou

Nanjing Medical University Affiliated Suzhou Hospital: Suzhou Municipal Hospital Jun Shen ( $D$ 18112603158@163.com )

Nanjing Medical University Affiliated Suzhou Hospital: Suzhou Municipal Hospital

\section{Research}


Keywords: Spine, Pedicle, Navigation, Imaging, Close range, Ultrasound

Posted Date: June 17th, 2021

DOI: https://doi.org/10.21203/rs.3.rs-617058/v1

License: (c) (i) This work is licensed under a Creative Commons Attribution 4.0 International License. Read Full License 


\section{Abstract}

Background: Ultrasound has been proved to be a promising alternative spine navigation method. High frequency ultrasound transducer has the advantage of high resolution on surface structure, but imaging at close range is difficult, especially in narrow space of the pilot-hole in pedicle.

Methods: Twenty cortical bone chips were made and different size of hole with diameter of $1 \mathrm{~mm}, 2 \mathrm{~mm}$, $3 \mathrm{~mm}$ or $5 \mathrm{~mm}$ was randomly carved in each bone chip. A tailored $30 \mathrm{MHz}$ high frequency transducer scanned bone samples at the distance of $4 \mathrm{~mm}, 3 \mathrm{~mm}, 2 \mathrm{~mm}$ and $1 \mathrm{~mm}$. Successive transmission ringingeffect elimination, Hilbert transform and Gray-scale mapping method were utilized to process and optimize attained original images.

Results: At the distance of $4 \mathrm{~mm}, 3 \mathrm{~mm}, 2 \mathrm{~mm}$ and $1 \mathrm{~mm}$, the holes with diameter of $5 \mathrm{~mm}, 3 \mathrm{~mm}$ and $2 \mathrm{~mm}$ could be discerned. At the distance of $1 \mathrm{~mm}$, only the holes with $5 \mathrm{~mm}$ and $3 \mathrm{~mm}$ could be clearly distinguished and the $2 \mathrm{~mm}$ hole appeared obscure. The holes with diameter of $1 \mathrm{~mm}$ could not be detected at any distance. The holes with diameter of $2 \mathrm{~mm}$ were able to be detected at the distance of $1 \mathrm{~mm}$.

Conclusions: This study indicated that the high frequency transducer had limited imaging capability at close range on the bony surface. These results lay a foundation for further developing a novel ultrasoundbased spinal pedicle interior imaging and navigation system.

\section{Full Text}

This preprint is available for download as a PDF.

\section{Figures}




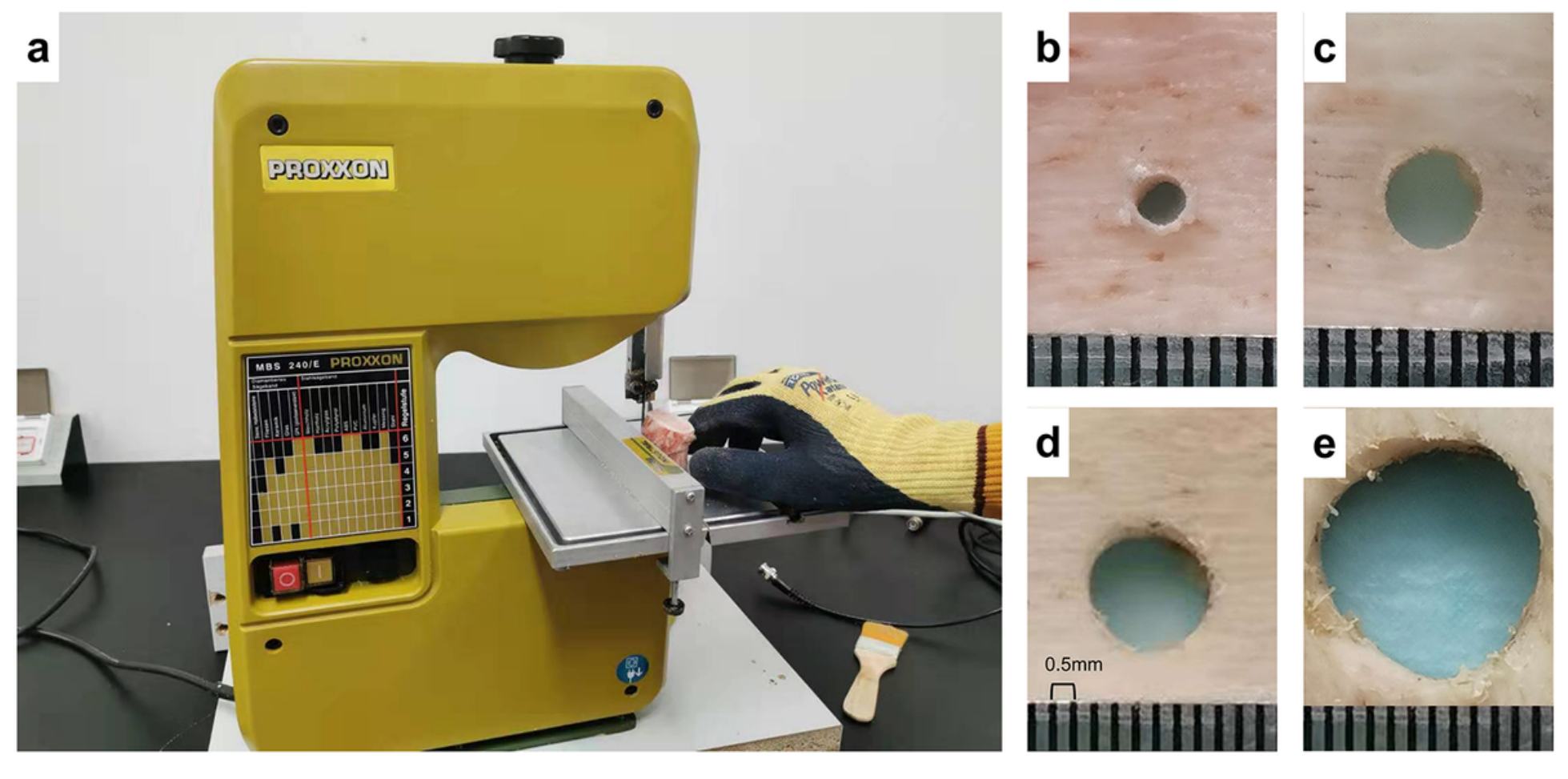

Figure 1

(a) The Chainsaw is used to fabricate the cortical bone chips. (b) The bone chip with diameter of $1 \mathrm{~mm}$.

(c) Diameter of $2 \mathrm{~mm}$. (d) Diameter of $3 \mathrm{~mm}$. (e) Diameter of $5 \mathrm{~mm}$. The scale of ruler is $0.5 \mathrm{~mm}$. 

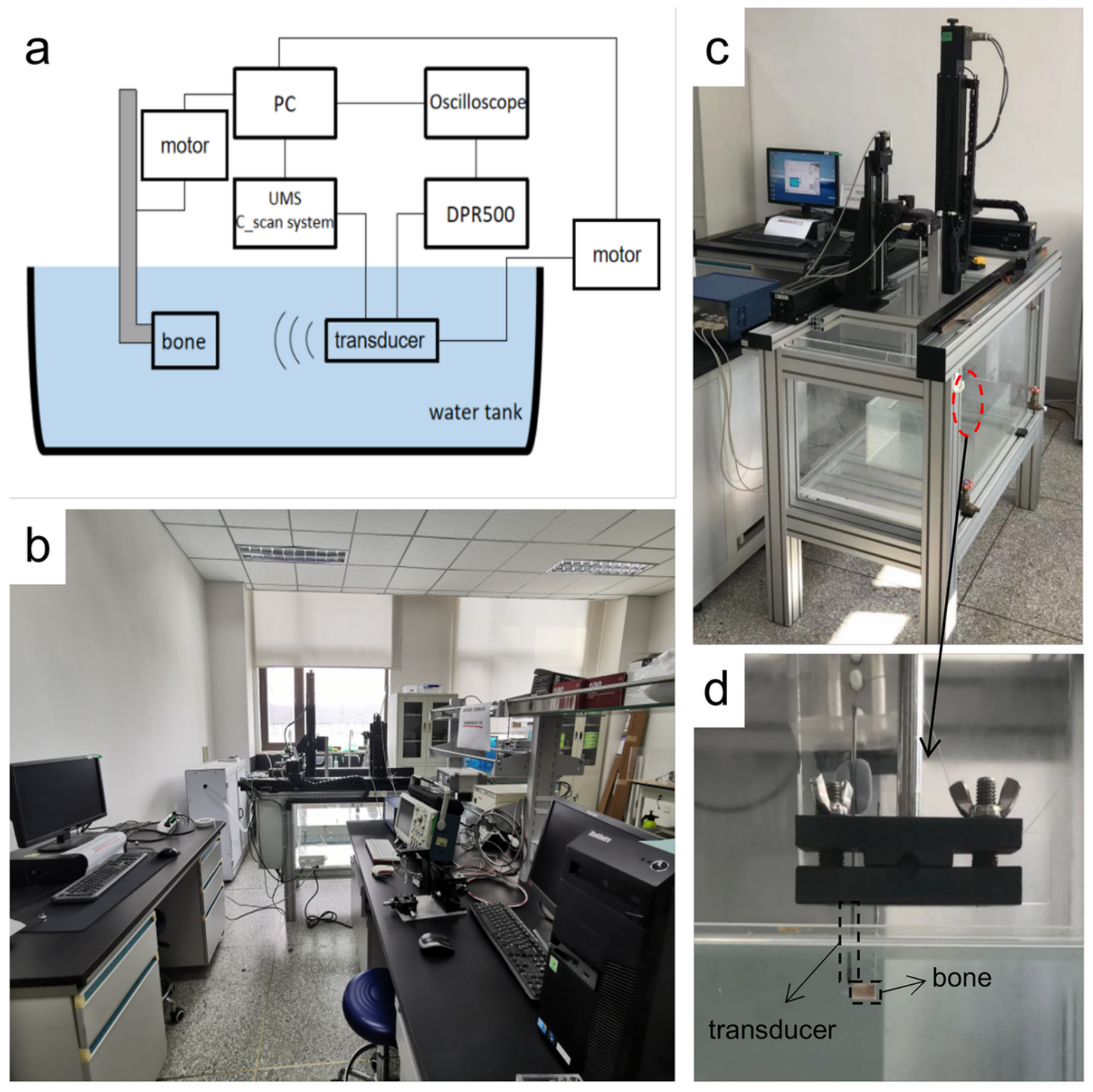

Figure 2

Experiment system used for ultrasonic detecting on cortical bone samples. (a) The schematic diagram. (b) Stereogram. (c) The bone specimen is fixed and measured by USM III. (d) Scanning is performed in the water bath. 


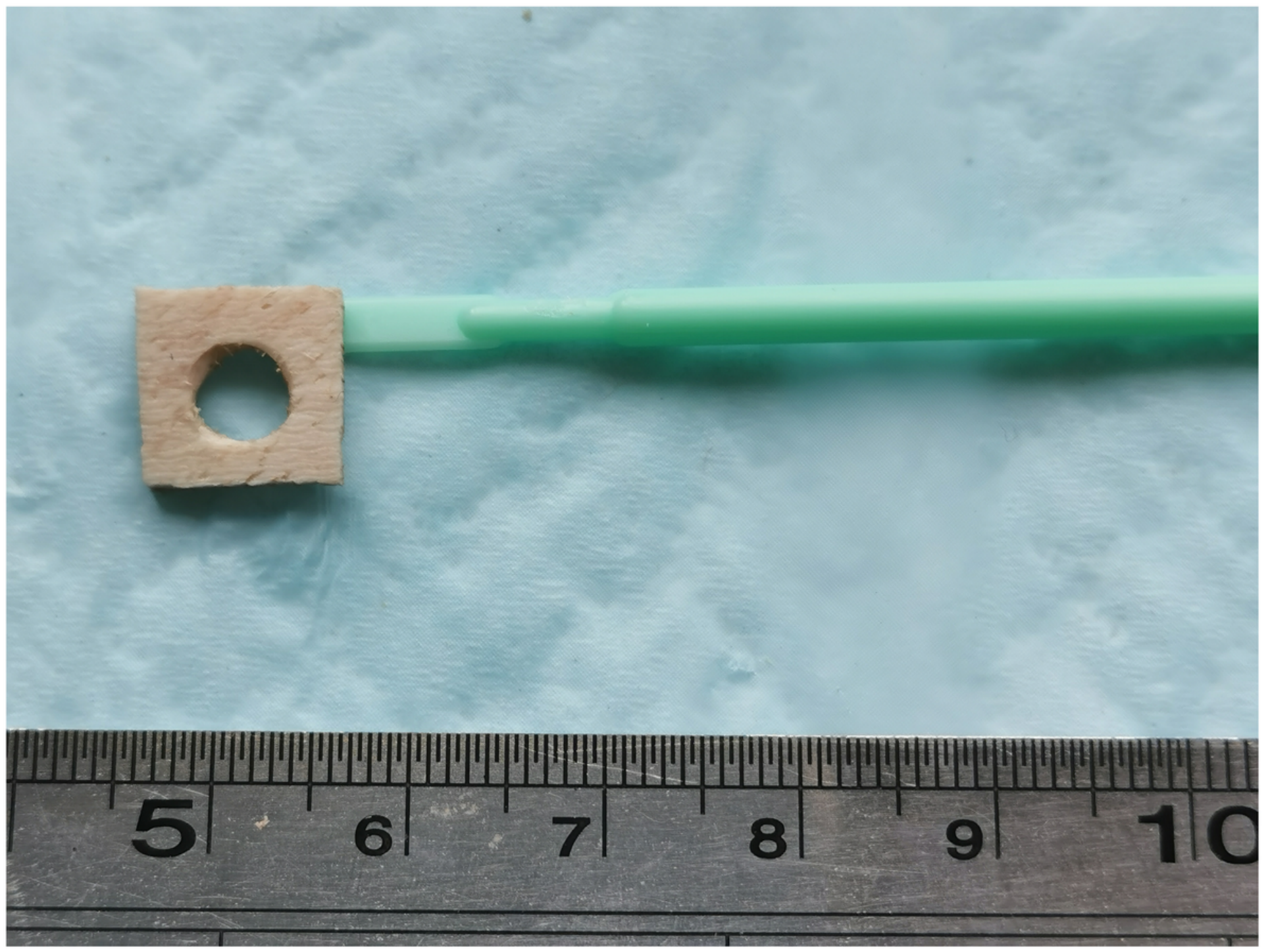

Figure 3

The ABS rod serving as a weak ultrasonic reflector is used to fix the bone sample. 
Distance

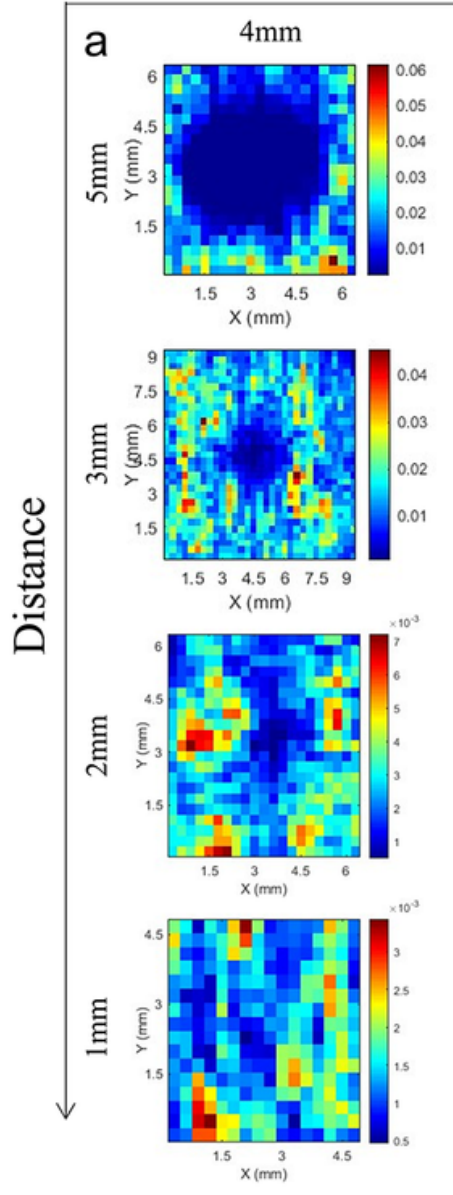

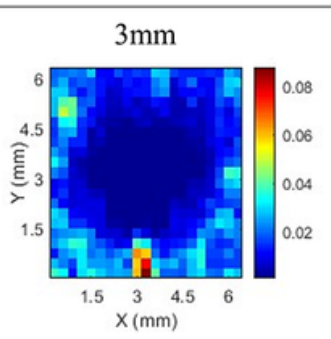
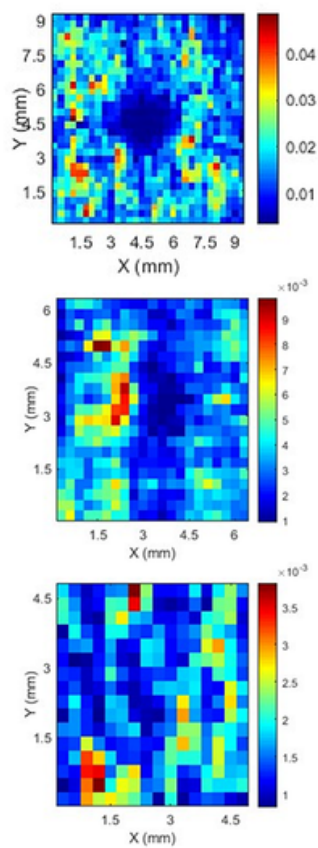
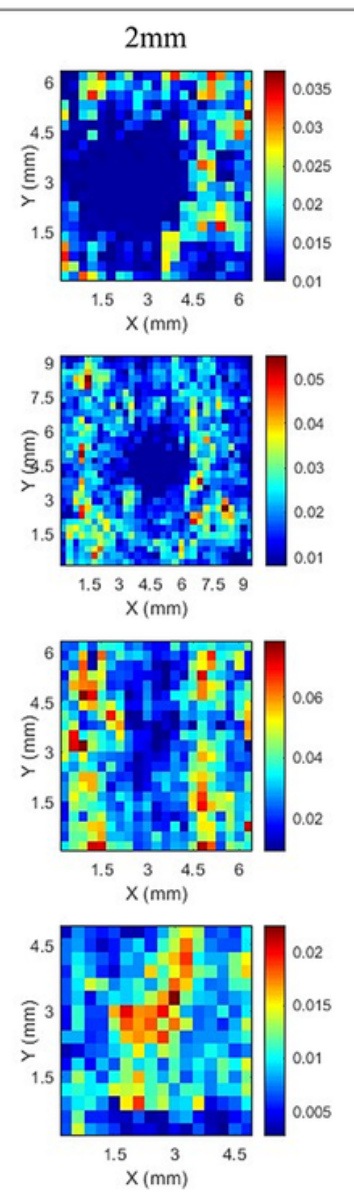
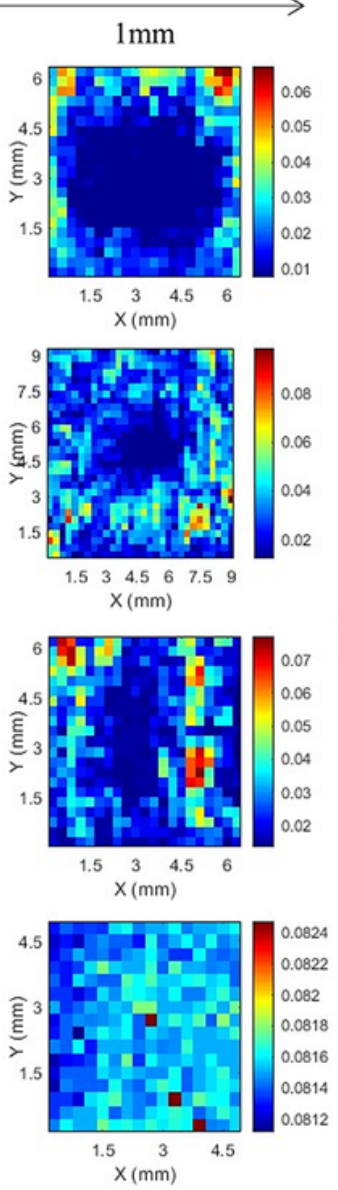

Distance
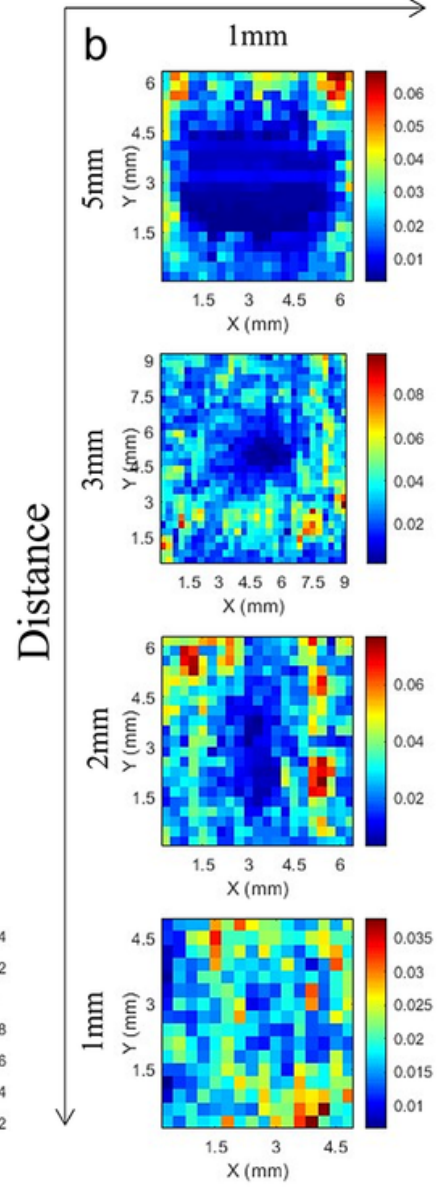

\section{Figure 4}

Original sound field images attained by $30 \mathrm{MHz}$ ultrasound transducer (a) The holes with diameter of $5 \mathrm{~mm}, 3 \mathrm{~mm}$ and $2 \mathrm{~mm}$ can be discerned at the distance of $4 \mathrm{~mm}, 3 \mathrm{~mm}, 2 \mathrm{~mm}$ and $1 \mathrm{~mm}$ from the transducer to the bone surface. The hole with diameter of $1 \mathrm{~mm}$ cannot be detected at any distance. (b) Further processed by the transmission ringingeffect elimination method, the hole with diameter of $1 \mathrm{~mm}$ still could not be discerned. (Each pixel of the generated image represents a $0.3 \mathrm{~mm} \times 0.3 \mathrm{~mm}$ square, from which we can simply estimate the dimension of the ultrasound image to be measured). 

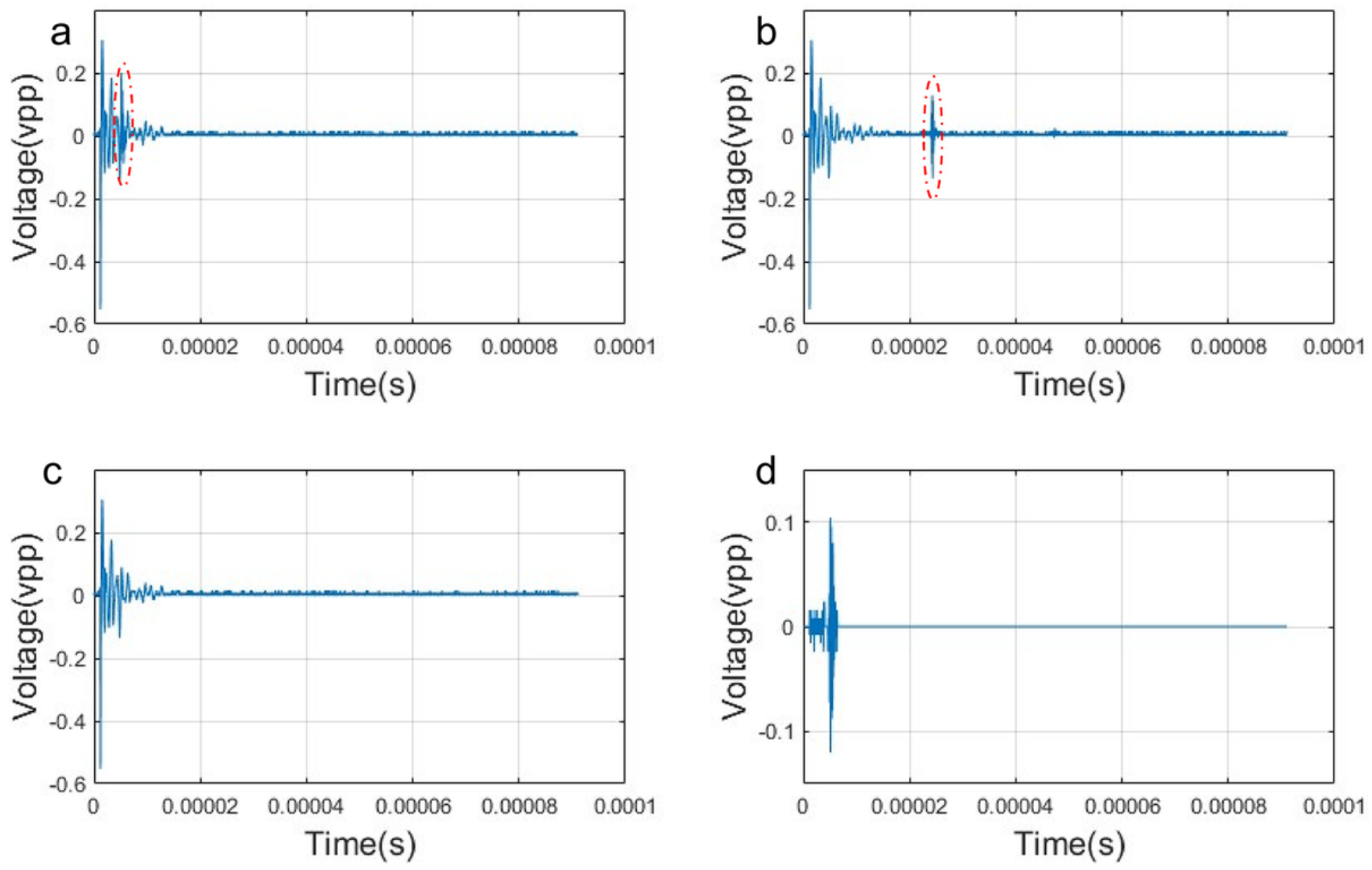

Figure 5

(a) The echo-signal is submerged in transmission ringingeffect signal (The pulse curve circled in red is the echo signal). (b) The echo-signal is outside of the transmission ringingeffect signal (The pulse curve circled in red is the echo signal). (c) Transmission ringingeffect signal. (d) The pure echo signal in Fig. 5(a) obtained by eliminating the transmission ringingeffect signal. 


\section{Distance}

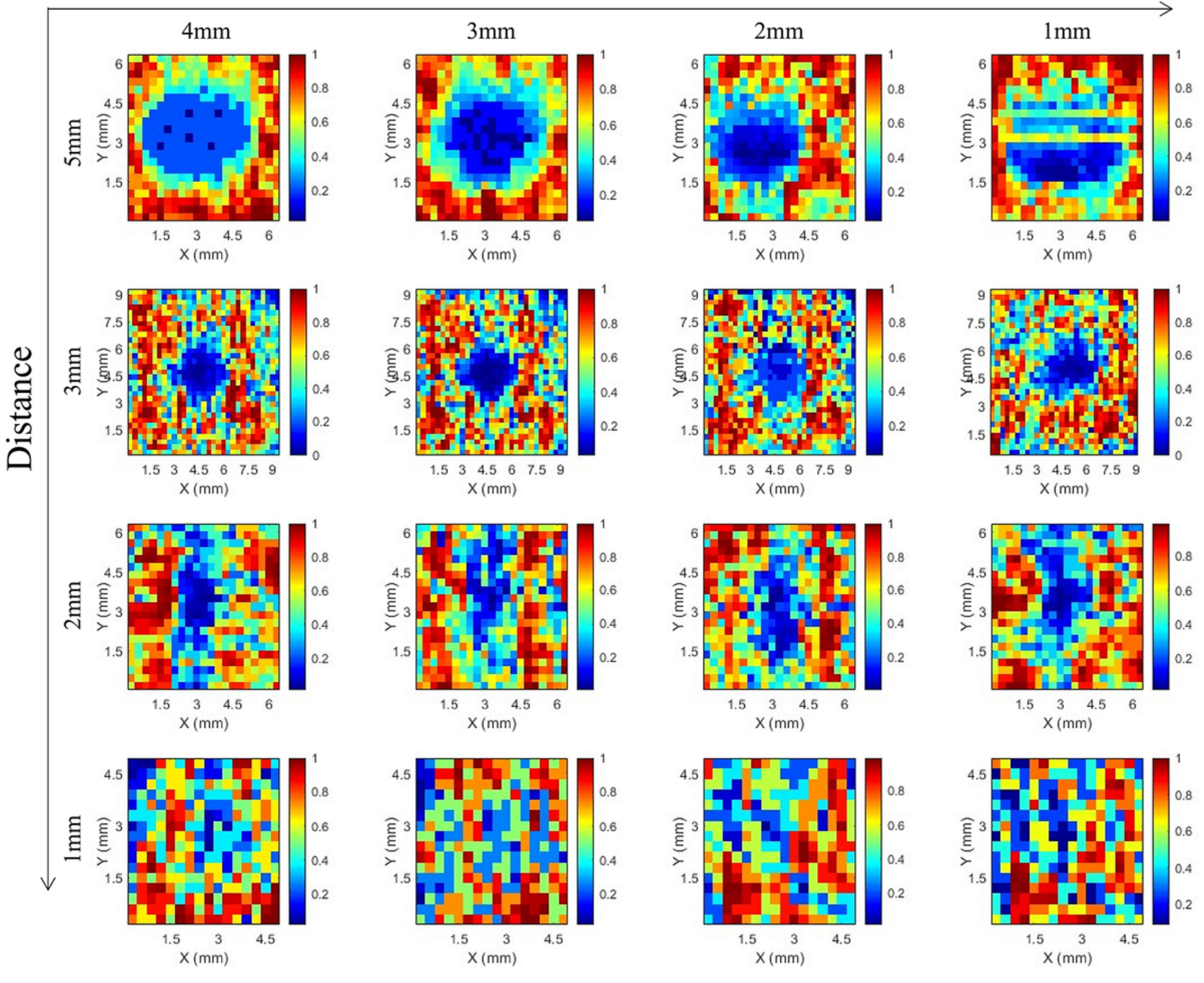

Figure 6

Hilbert transform and logarithmic function based gray-scale mapping method are sequentially utilized to achieve B-mode images. The images of the holes with diameter of $5 \mathrm{~mm}, 3 \mathrm{~mm}$ and $2 \mathrm{~mm}$ are clearer than before, and the hole with diameter of $1 \mathrm{~mm}$ still cannot be detected at any distance. 

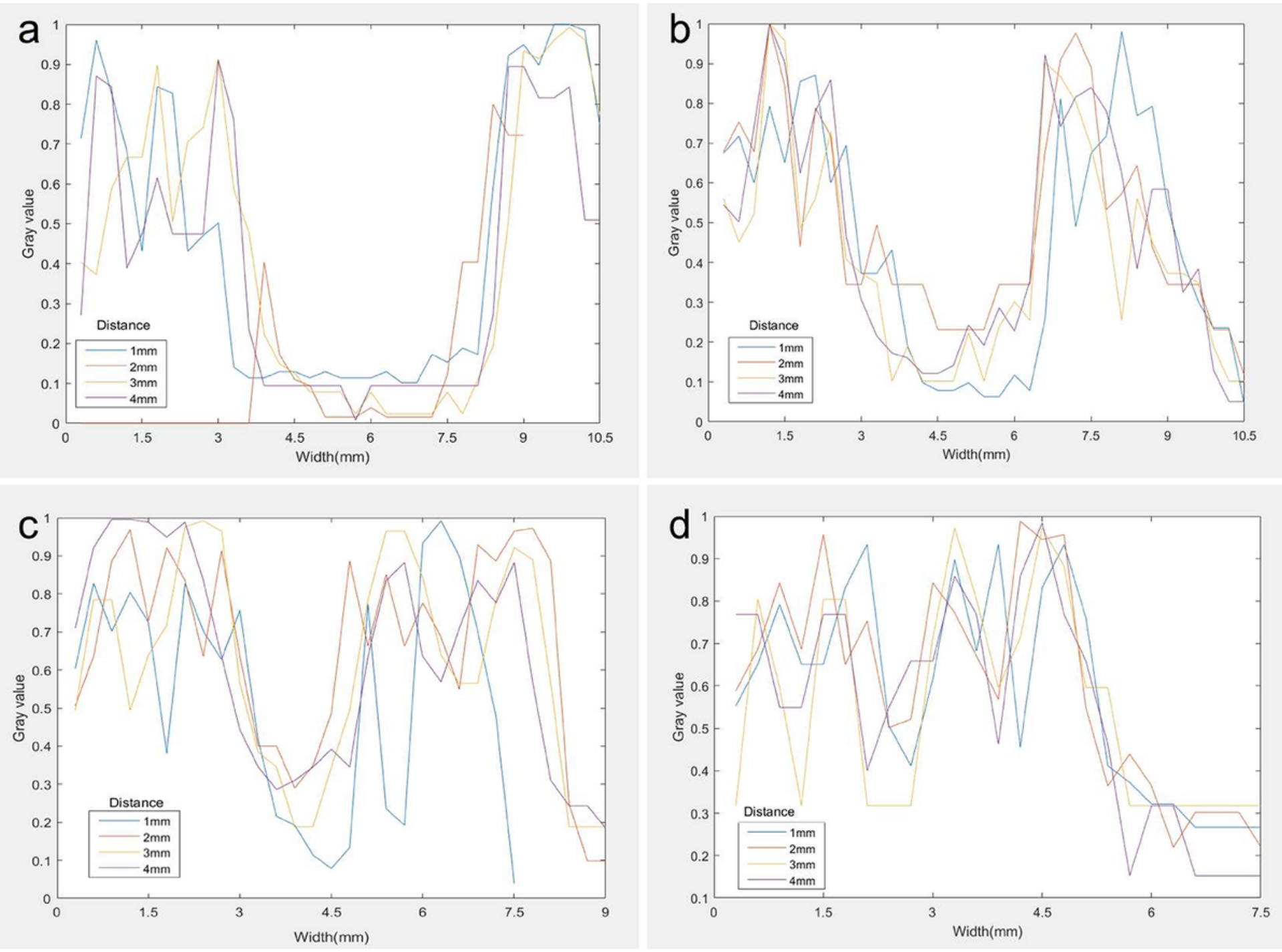

\section{Figure 7}

The four sizes of holes are successively detected at the distance of $4 \mathrm{~mm}, 3 \mathrm{~mm}, 2 \mathrm{~mm}$ and $1 \mathrm{~mm}$. (a) The hole with diameter of $5 \mathrm{~mm}$. (b) The hole with diameter of $3 \mathrm{~mm}$. (c) The hole with diameter of $2 \mathrm{~mm}$. (d) The hole with diameter of $1 \mathrm{~mm}$.

\section{a}

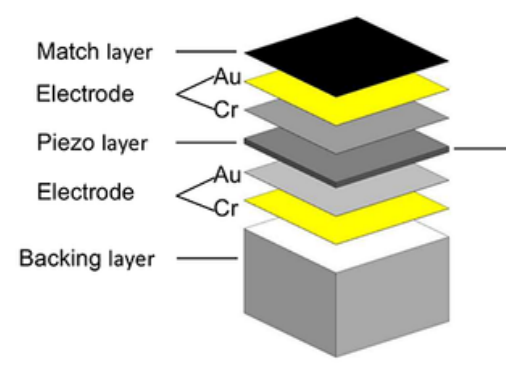

b

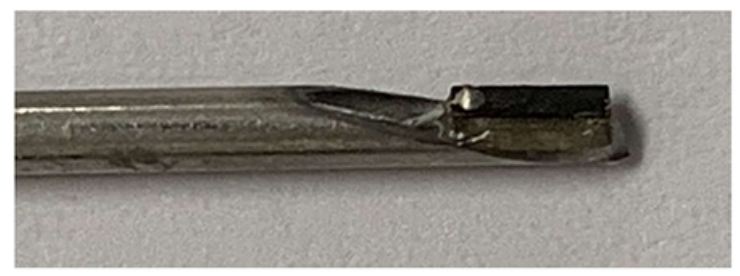

\section{Figure 8}

The schematic diagram of $30 \mathrm{MHz}$ fabricated transducer (a) The three-tiered structure. (b) Single-element ultrasonic transducer of $30 \mathrm{MHz}$. 


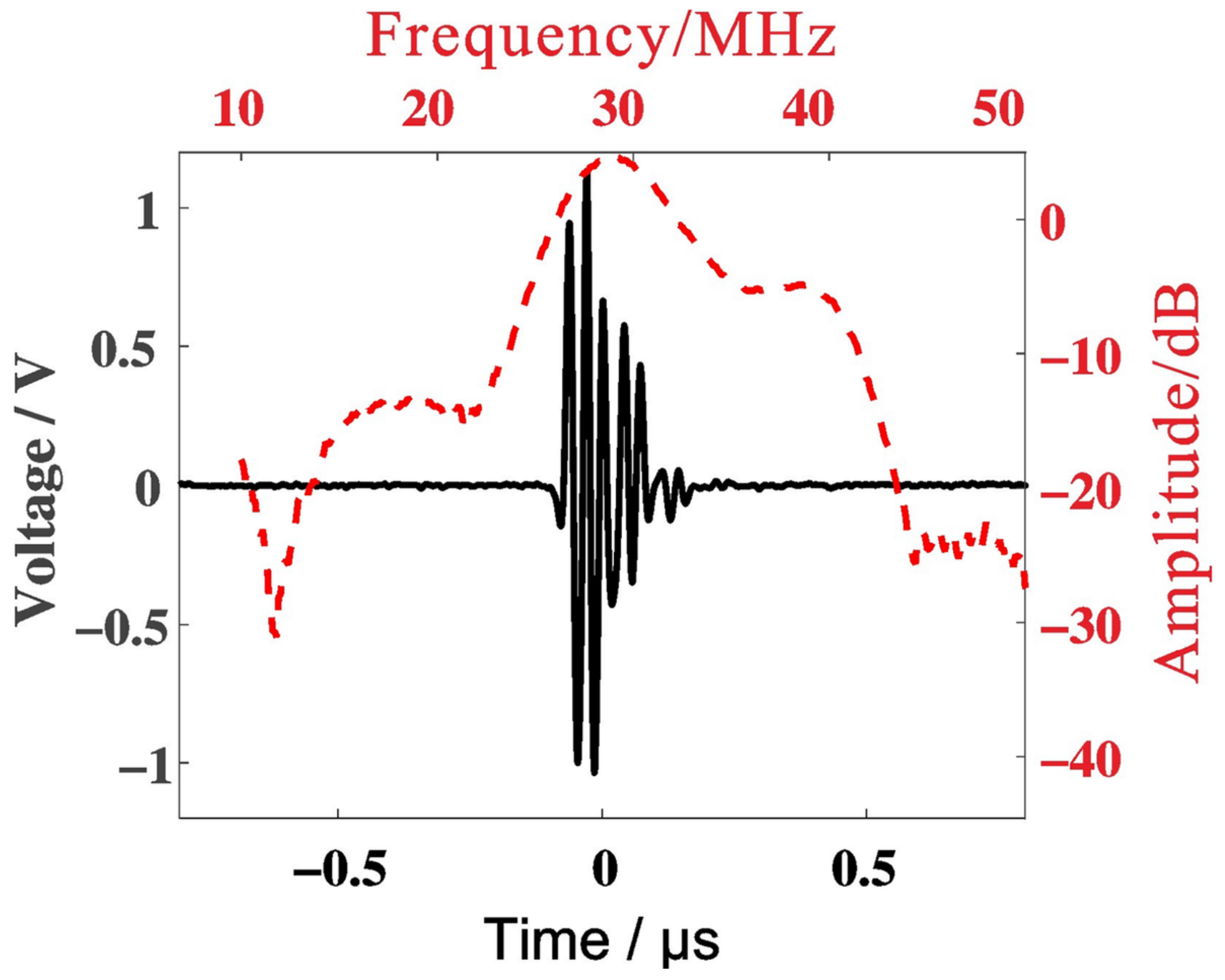

Figure 9

The pulse-echo curves of the transducer. The radio frequency (RF) curve is shown in the black line and the envelope curve is shown in the red line. 